\title{
HACIA UN MODELO EDUCATIVO INTEGRAL QUE HUMANIZA
}

\author{
Jorge Flores Silva , Universidad Nacional Autónoma de Honduras, \\ Instituto de Investigaciones Económicas y Sociales (IIES UNAH), \\ Ciudad universitaria edifico c2 primer piso. Tel./Fax: 504-22391849 \\ Correo electrónico: jflores@iies-unah.org
}

\section{RESUMEN}

Un modelo educativo con calidad y equidad ha de estar en función de la vida. Vivir y saber convivir debe ser tarea fundamental en toda propuesta educativa, porque la educación también es propuesta ética en el sentido que humaniza y socializa.

Pensar y saber pensar para la reflexión que oriente a la transformación de las realidades que excluyen y deshumanizan debe ser resultado de procesos humanos que privilegian él "para que" y no solo el "como" de las cosas. -En tal sentido el curriculum que se impulse como campo problemático, complejo y abarcador debe ser de carácter integral que incluya los contenidos conceptuales, procedimentales y actitudinales para formar un ciudadano con sentido de responsabilidad y con conciencia del sujeto. Buscar la verdad es el objeto propio del deseo de educarse, verdad que se busca en la cultura, la economía, la política y en las relaciones interpersonales. La universidad es un espacio propicio para ello, donde no solo se forman profesionales, sino también donde se crea ciencia y se valora el trabajo científico con sentido de pertinencia que va al descubrimiento o que busca alternativas de solución a problemas concretos de la sociedad.

Palabras clave: Globalización, Curriculum, Ética, Epistemología, Ciencia, Pertinencia, Educación. 


\title{
TOWARDS A COMPREHENSIVE EDUCATIONAL MODEL THAT
} HUMANIZES

\author{
Jorge Flores Silva , Universidad Nacional Autónoma de Honduras, \\ Instituto de Investigaciones Económicas y Sociales (IIES UNAH), \\ Ciudad universitaria edifico c2 primer piso. Tel./Fax: 504-22391849 \\ Correo electrónico: jflores@iies-unah.org
}

\section{ABSTRACT}

An educational model with quality and equity must be a function's of life. Live and learn to live should be fundamental task in any educational proposal, because education is also ethical proposal in sense that humanizes and socializes.

Keywords: Globalization, Curriculum, Ethics, Epistemology, Science, Relevance, Education. 


\section{INTRODUCCIÓN}

Generalmente, cuando se habla del problema de la educación, se habla de reformar la educación básica, la educación secundaria y muchas veces no se le da prioridad a la educación universitaria. Es la universidad la que tiene que llevar un efectivo liderazgo. Los resultados de la educación superior dependen de los resultados de la educación media y es en la universidad donde se prepara a los profesionales que van a ir a preparar a los educadores del nivel medio, es en la universidad donde se diseñan los instrumentos, se preparan los textos, se prueban las técnicas metodológicas, por eso, la universidad tiene que jugar un papel muy importante y ser líder en el impulso de un nuevo modelo educativo en función de un nuevo modelo de desarrollo.

Al hablar de un modelo educativo se ha de hablar del curriculum como campo complejo y abarcador. Muy a menudo se habla de calidad de la educación pero cuando se habla de calidad no debe hablarse solo de eficiencia y eficacia, también hay que hablar de pertinencia en el sentido de que sea útil no solo a un sector, sino a toda la sociedad-, pero que es pertinente para nosotros, que es pertinente para el aparato productivo en su conjunto en fin que es pertinente para los sectores más empobrecidos de la sociedad. Todo ello hay que considerarlo en un nuevo modelo educativo.

\section{EDUCACIÓN Y ECONOMÍA TAREAS COMPLEMENTARIAS}

La educación no solamente es transmitir conocimientos, no solamente es informar, sino formar; como lo señalan los clásicos de la educación; implica desarrollo intelectual, implica el aspecto psicobiosocial, la parte de la personalidad humana, la parte que responde al hacer y al pensar, para qué hacer y cómo aplicar, incluyendo la parte espiritual y la concepción que tenemos de la sociedad y el mundo incluida la formación física o corporal que tenemos para desarrollar la actividad productiva. Es así que se necesita formar el individuo de manera integral por tanto no se puede concebir un curriculum fragmentado.

Con frecuencia se habla de reformas en la educación, se llevan a cabo foros y debates pero nunca se llega a realizaciones prácticas. Es necesario pasar del discurso a la acción. La reforma educativa y la reforma de la estructura productiva; los estilos de vida y los modos de consumir no son independientes.

No se puede salir de la pobreza solamente con hacer la reforma educativa; muchas veces el padre de familia está en la disyuntiva entre mandar a sus hijos a la escuela 
o mandarlos a trabajar para que contribuyan al sustento familiar. La problemática ha de abordarse de manera simultánea porque no solo es necesario educar para salir de la pobreza, sino que también el problema de la pobreza hace que la población no se eduque; es decir hay una interrelación, y se puede generar un circulo vicioso o resolvemos un problema, o resolvemos el otro, o hacemos una actividad simultanea pensando hacer los cambios en función de todos.

El estado tiene que cubrir parte de la educación del pueblo a través de los impuestos, porque la educación inicialmente nos da un beneficio personal, pero con nuestra educación se beneficia toda la sociedad; la educación de un profesor le da un beneficio personal o recibe una remuneración por ese servicio concreto que presta, pero también se beneficia la sociedad a la cual está educando, lo que los economistas Ilaman las externalidades o sea la ventaja social. Es decir con la educación nos beneficiamos todos. Por eso es que la educación tiene que financiarla el estado, que generalmente lo hace con los impuestos aunque una parte la financie el interesado. En un modelo de la máxima ganancia en función del interés privado, la educación resulta inequitativa porque el que tiene un buen nivel de ingresos puede ir a una escuela donde hay adecuadas instalaciones, tienen laboratorios bien equipados, bibliotecas, áreas para hacer deporte y expansión humana, etc. en tanto que la educación pública sufre de muchas carencias o no tiene todos esos servicios, y de ahí viene la inequidad en la educación.

Como reflejo del modelo económico que se promueve, se quiere con exclusividad someter la actividad educativa al aparato productivo, siendo las actividades mecánicas las que se utilizan como elemento de evaluación para decidir si los conocimientos y el desempeño de los egresados de las instituciones de enseñanza son validos, es decir reducir la noción educativa a una esfera de la personalidad relacionada con habilidades técnico-motrices, y no en su verdadera dimensión psico-biosocial que prepara al individuo para la vida y su interrelación en la sociedad de la cual forma parte. Este modelo de educación eficientista es el que consciente o inconscientemente, se impulsa y promueve.

Es tiempo de formar a los educadores, sobre los cuales estará la mayor responsabilidad de dirigir los procesos pedagógicos, bajo una concepción científica de la educación, en donde el sujeto sobre el cual recae la acción educativa, se forme para tener una visión crítica del mundo que le rodea, para emprender la acción transformadora en una sociedad cada vez más dinámica, compleja y llena de incertidumbre.

La multiplicidad de fenómenos tienen implicaciones económicas, en tal sentido, al 
platearse un modelo educativo, o una reforma educativa cuyo eje central sea la calidad, mediante el diseño de un curriculum que incluye además de los saberes y el alumno, también el entorno socioeconómico, entonces no se puede negar que será necesario considerar para cualquier disciplina la dimensión económica, que permita desde luego articular la demanda socioproductiva, con la oferta profesional, no pensando únicamente en la eficiencia económica en función del interés particular que excluye a los más débiles. Entonces se ha de preparar un ciudadano no solo para obtener un empleo o ir a realizar actividades de rutina, sino un individuo con capacidad de discernimiento, reflexión y análisis, con mentalidad abierta y actitud positiva para ser cada día mejor, un ciudadano identificado con el país, que no subestime los valores nacionales, ni siga creyendo que todo nos vendrá de afuera para resolver nuestros problemas. Con frecuencia se escucha decir que los egresados de los centros educativos en los distintos niveles y para las diferentes disciplinas son muy teóricos, esto refleja que el empleador solo valora, los elementos procedimentales relacionados con habilidades técnico-motrices, menospreciando en el profesional lo conceptual y actitudinal propios de una persona con formación integral, que forma parte de un contexto y está dotado de un esquema de valores e identidad propia, capaz de discernir y adaptarse a situaciones cambiantes. Ello es así porque el aparato productivo que demanda fuerza de trabajo, se aparta de la complejidad y la investigación, solo exige la realización de trabajos que requieren procesos propios de un esquema mecanicista, autómata y carente de toda iniciativa que estimule la creatividad y la innovación.

De ahí que se puede deducir que la escasa formación profesional de la que tanto se acusa en forma indiscriminada a todos los egresados de los centros de educación superior solo es una forma de reconocer que el potencial teórico y practico de que disponen, no está en correspondencia con lo que demandan las empresas, porque los requerimientos de estas son tan limitados que evitan la contratación de personal con fuerte preparación académico-científica.

La institución educativa particularmente las universidades deben seguir siendo espacios para el análisis y el debate académico, la investigación y la práctica científica donde se construye el conocimiento. Deben ser instancias para la promoción de valores humanos, la difusión de la cultura, la democratización política, el respeto a los derechos humanos y la convivencia armónica, asumiendo un papel protagónico en la solución de problemas que afectan a la sociedad, evitando caer exclusivamente en el curriculum tecnocrático que obedece también a un modelo excluyente que subestima la creatividad y las potencialidades 
humanas, principalmente de los que están fuera de las relaciones de mercado.

Tan importante es la formación técnica, como la formación humana, que se dará al individuo en el proceso de enseñanza-aprendizaje, pero más importante es saberlas armonizar en función de las necesidades, intereses y aspiraciones de la sociedad para que esta formación tenga el verdadero significado social.

En un mundo marcado por la globalización económica, no quiere decir que se ha de pensar solo en la inserción exitosa al proceso de globalización desde la perspectiva de la "calidad, la eficiencia y la competitividad" en función del utilitarismo para el aparato productivo y las exigencias del mercado. La educación no puede seguir subordinada a los valores supremos del mercado que excluye y margina, hay que revertir este modo de ver la educación y la economía, la educación debe ser una vía por la cual transita la incubación de una conciencia crítica que derive y conduzca a una transformación de la realidad, en beneficio de los ciudadanos.

Las instituciones, en tanto que son instancias que expresan las políticas y objetivos, tanto del sector público, como de la sociedad civil, así como las personas que entrañan los esfuerzos institucionales para llevar a cabo las mencionadas políticas, están llamadas a centrar el desarrollo económico y social en el desarrollo de la persona. La ética juega un papel importante, sin ética no se puede vivir, la ética postula como criterio básico la dignidad de la persona, centrar el desarrollo en el desarrollo de la persona, es tarea fundamental en una política de desarrollo

\section{EDUCACIÓN DE CARÁCTER INTGRAL Y CON EQUIDAD}

La educación debe ser instrumento socializador que humaniza, por eso los curriculums que se impulsen debe ser de carácter integral que incluyan los contenidos cognitivos, procedimentales y actitudinales. Lo conceptual nos lleva al conocer, lo procedimental al hacer y el actitudinal al ser. Desde esta perspectiva la educación no solo debe tener un enfoque utilitarista, educación y economía deben complementarse. Además la educación debe cultivar una conciencia que impida seguir actuando arbitrariamente, en relación a los recursos naturales y a sus semejantes.

La educación como práctica social debe ser de carácter universal, y no reducida a determinados sectores. Debe brindarse con equidad sin exclusión y con calidad para todos. El pobre tiene iguales derechos que el rico, el uno es tan ciudadano como el otro, en ese sentido todos tienen derecho de una educación de calidad 
excluye a unos o se brinda distinto tipo de educación se cae en la injusticia.

La educación en los países subdesarrollados, de la cual Honduras forma parte, todavía sigue siendo un privilegio para ciertos sectores de nivel socioeconómico alto, factor que se expresa en la inequidad tanto en el plano cuantitativo como cualitativo. No se puede hablar de equidad, ni mucho menos de calidad educativa, cuando todavía hay estudiantes que reciben sus clases sentados en el suelo, que no pueden articular la teoría y práctica científica porque carecen de laboratorios. Tampoco hay lugar para la expansión humana porque los reducidos espacios no les permiten la realización de algún deporte como parte de su formación integral.

Se desarrolla una educación que está en crisis, que resulta en crisis de las ideas porque es la misma sociedad que está en crisis, he aquí un elemento clave en la calidad de la educación. Generalmente los indicadores de medición para calificar la educación se circunscriben a los índices de matrícula, deserción y repitencia, sin tomar en cuenta que la sociedad demanda de un enfoque valorativo para juzgar y actuar.

En la universidad se privilegia más la docencia que la investigación, hay mucha docencia y poca investigación. Todavía no se alcanza a comprender que las universidades son instituciones de desarrollo en las que se debe crear ciencia. La universidad es el espacio propicio para articular las funciones básicas de docencia e investigación, al tiempo que se formulan efectivos programas de vinculación con la sociedad. Conviene entonces desarrollar la investigación básica que nos lleve al descubrimiento y así acrecentar los conocimientos de la realidad observable. También es fundamental impulsar la investigación aplicada para platear alternativas de solución a problemas concretos de la sociedad y el individuo.

El mundo actual y el modo de funcionar de la economía demanda de altos niveles de formación profesional para participar en el complejo campo de las relaciones personales, sectoriales e interfactoriales. Asimilar los avances de la tecnología y procesos de información rápida exige de estos altos niveles de preparación que permitan adaptarse a la era de la complejidad y la incertidumbre para tomar las mejores decisiones.

La práctica educativa a través de los distintos medios que orientan el camino para llegar al conocimiento, no solo permite orientar la enseñanza, también permite transmitir valores para la convivencia social. Cuando la educación privilegia el "ser" por encima de lo tecnocrático, cuando se da prioridad al conocimiento y no a lo instrumental, cuando el curriculum es de carácter integral que no descuida ninguna de las áreas en el campo biopsicologico, entonces la educación cumple su 
papel de realizar la personalidad, esta educación para el bienestar personal, para vivir mejor y para el desarrollo, debe ser siempre apoyada.

Saber comunicarse para la búsqueda y el descubrimiento es factor indispensable en la construcción de los sistemas teóricos de la ciencia. Para que haya creación de ciencia se necesita del científico, el profesor docente sobre todo el de educación superior debe buscar este perfil porque es en las universidades donde se forman los que han de formar a los formadores, los científicos, los administradores y los que han de dirigir los destinos de la sociedad.

La educación como disciplina no es neutra siempre permite tomar postura en la práctica de la enseñanza, para dar respuesta a los problemas del desarrollo. En la sociedad el ser humano es el motor central del crecimiento y el desarrollo, para ello debe estar bien capacitado y educado de manera que pueda enfrentar y confrontar las vicisitudes de la vida e insertarse a la transformación de cara a las exigencias de la globalización.

La pluralidad científica y epistemológica es de indiscutible valor en la educación, sobre todo reconocer el auxilio que dan a las ciencias de la educación la filosofía, la psicología, la antropología y las ciencias sociales en general para lograr esa pluralidad.

Fomentar el análisis y la reflexión crítica, así como el de cultivar cualidades éticas, intelectuales y afectivas para sí y para los alumnos sujetos del aprendizaje demandan un buen perfil docente, el cual es adquirido con una solida formación técnico-científica que incluye formación pedagógica, formación didáctica y formación en su propia disciplina. Esta formación de los que han de formar ha de significar que el profesor procure que el alumno, aprenda hacer, sepa aplicar, y sepa convivir sabiendo ser. De ahí que la educación y el acto docente deben ser una necesidad sentida para lograr el perfeccionamiento y la excelencia académica.

La educación es una ciencia ideográfica, sobre la misma hay diversas percepciones y distintos modos analíticos del proceso de construcción del conocimiento. Corresponde a los educadores la responsabilidad de diseñar y poner en práctica un modelo educativo que responda a las necesidades nacionales, que plantee alternativas de solución educativa para lograr las aspiraciones más sentidas de los ciudadanos, aspiraciones que no solo obedecen a lo tangible sino que incluye aquello que no se palpa, al mundo de las ideas, de las creencias y que muchas veces son las necesidades más sentidas. Sociedades subdesarrolladas como la hondureña, mantienen sistemas educativos subdesarrollados porque aunque se 
han impulsado modelos que han intentado modernizar el sistema, estos más bien han tenido una visión tecnocrática. Más aun la pertinencia se concibe como la forma en que se ha de responder a las exigencias de la economía global, al utilitarismo del aparato productivo y a la necesidad de satisfacer la maximización de la ganancia en función del interés particular por encima del interés social.

La lógica económica del modo de funcionar de la economía ha orientado el sistema educativo. Ahora hay más preocupación por lo técnico e instrumental que por el conocimiento, no se puede trasladar en forma mecánica el modelo de calidad total para la eficiencia del sector privado, al sistema educativo como gestor de la educación de calidad. Es la educación en un contexto de interdependencia y de relaciones factoriales e intersectoriales que debe diseñar un curriculum educativo para la relevancia y la pertinencia en la que se combine la formación humana con la técnica, para promover la auto reflexión crítica y la efectiva participación ciudadana con espíritu de solidaridad y colaboración, sabiendo que la educación tiene sentido social y está llamada a hacer el bien a la comunidad.

La educación como generadora de procesos humanos, influye en las distintas esferas de la personalidad, llegando a modificar pautas de comportamiento. El ser humano por su propia naturaleza está expuesto a conflictos, en tal sentido cualquier diseño curricular debe incluir la orientación y consejería para atender las necesidades personales de los educandos. No debe descuidarse tampoco lo relativo al uso de los libros de texto por cuanto estos llegan a ser instrumento de control del curriculum. Muchas veces los textos utilizados en el proceso $d$ enseñanza-aprendizaje distorsionan la realidad o tienden a impedir la reflexión y el análisis en función de diferentes enfoques o perspectivas. La educación y sobre todo la superior demanda de un carácter universal que debe estar abierta a las distintas corrientes de pensamiento, por ello la universidad está llamada a ser no solo formadora de profesionales en donde se privilegia el acto docente en desmedro de la acción investigativa orientada a crear ciencia. La universidad es una institución de desarrollo que debe ser creadora y productora de libros, el pensamiento crítico debe darse a conocer en revistar sin perder la identidad, esta función de crear ciencia y desarrollo de la reflexión y el debate debe convertir a los actores principales del quehacer académico en intelectuales atentos a los nuevos tiempos, desconfiados frente a cualquier sistema globalizante que excluye, margina y deshumaniza.

La educación como proceso de construcción del conocimiento no es independiente de la filosofía de la ciencia que demanda la reflexión sobre el 
conocimiento científico. La búsqueda y el descubrimiento es tarea educativa, los resultados deben llevar a la solución de problemas y al debate para profundizar en el análisis del sentido de la acción. Solo la acción transforma la realidad. Para transformar la realidad se necesita conocerla, ello implica el diagnóstico resultado de un proceso educativo que permite conocer la problemática y el proceso histórico que generó esa problemática. Educar no solo es transmitir, sino también crear. El estar educando no solo está relacionado con el conocer, el hacer o el saber convivir para ser; necesita también saber descubrir.

La educación debe preparar para los grandes propósitos, con relación al otro, consigo mismo y con el bien común. La educación debe estimular la auto promoción, pero al mismo tiempo debe tener sentido social. Deja de ser educación incluyente cuando se promueven y diseñan modelos educativos individualistas, curriculum fragmentados y tecnocráticos que privilegian lo técnico por encima del conocimiento. La autorrealización plena se dará en la medida que se consideren los intereses generales de la sociedad, teniendo como fundamento el otro.

\section{HACIA UN PROYECTO EDUATIVO DEL DEBER SER}

E\&A IIES

El discurso en las ciencias de la educación debe dar orientación a partir de una plataforma epistemológica que defina con claridad teórica y metodológica el tipo de educación que demanda la sociedad. No se puede seguir hablando en forma vaga, general y ambigua de calidad de la educación, crisis de la educación y democratización de la educación. La educación es una ciencia sobre la cual hay distintas concepciones, como hay distintas percepciones de la realidad, así también hay distintas concepciones sobre democracia y sobre lo que se puede entender por calidad de la educación. Se puede hablar de democracia siempre y cuando no afecte los intereses de grupo, el estado de cosas o la institucionalidad del sistema, también se puede hablar de calidad siguiendo la lógica del modelo predominante. En educación la calidad puede verse desde distintas perspectivas: calidad metodológica, calidad social, calidad política, según sean los objetivos que se persigan en el diseño curricular. Estos objetivos y propósitos son definidos según la visión que del mundo y la sociedad tengan sus impulsores. Generalmente las políticas económicas son orientadoras de la política educativa, al menos esa ha sido la experiencia hasta ahora. En una sociedad regida por sectores con distintos intereses será difícil congeniar con un proyecto educativo único que satisfaga las aspiraciones de todos. No es fácil llegar a un consenso con grupos que tienen distintas expectativas y esperan resultados de la acción educativa según sus 
intereses.

La buena educación demanda de un buen sistema educativo, que formule iniciativas para el diseño curricular. Esto pasa por la formación de buenos maestros con vocación de servicio, sin descuidar la buena dirección, la supervisión y la buena administración académica.

El acto donde empieza por saber comunicarse con los estudiantes de manera que estos puedan disfrutar del proceso de búsqueda y adquisición del conocimiento. En esto el educador no solo debe preguntarse ¿Qué enseñar? A partir de la respuesta que se dé a esta pregunta se podrá saber si se está brindando una educación con pertinencia.

La pertinencia no debe verse en sentido reduccionista que lleva al individuo a realizarse solo con su propio conocimiento sin pensar en la interdependencia para darle un enfoque social. Tampoco debe caerse en la falsa idea de que la buena educación y la pertinencia debe medirse por lo que pueda darle al aparato productivo.

La educación debe considerar los valores en el curriculum integral. Desafortunadamente no siempre se toma en cuenta los valores porque no forman parte del mundo objetivo. Hay valores científicos que demandan de la evidencia argumento para solo considerar el valor de la verdad desde la perspectiva cientificista, que considera a los demás valores como subjetivos, por ello se soslayan del curriculum educativo. La educación como forma de influir en el comportamiento humano, en cierta medida causa preocupación porque puede ser el medio para cambiar el status quo, por eso no se da énfasis ni se incluye el esquema de valores de manera prioritaria en el proceso enseñanza-aprendizaje que forma al individuo para que participe en los procesos de transformación. Esta visión que huye de los demás valores, considerados como parte de las virtudes humanas o del deber ser es claramente tecnocrática y racionalista.

Todo se juzga por lo que se ve y se palpa, prepara al individuo para hacer cosas. Una educación integral no puede valorar solo el conocimiento o el cómo hacer, educarse es prepararse para la acción y el ejercicio de los derechos humanos, es desarrollar las capacidades y potencialidades humanas para la construcción de una sociedad más fraterna, mas soberana y más solidaria. Desde esa perspectiva la educación no puede dejar de lado el valor de la justicia, la solidaridad, el respeto a la ley, la cultura y todo aquello que valora al ser humano por lo que es y no por lo que tiene. Estos valores son elementos constitutivos para trabajar por el bien común. Descubrir las virtudes del individuo y desarrollar sus capacidades humanas 
es fundamental en el diseño, construcción y ejecución de un curriculum educativo.

Educar para pensar debe ser un objetivo en todo proceso de enseñar y aprender. La motivación intrínseca del ser humano de saber es la que lo lleva a participar en el acto educativo. Esta tarea se encamina a comprender, a conocer, asimilar y no solo hacer.

Educar a los pobres, desburocratizar el sistema, favorecer lo cognitivo y actitudinal sobre lo técnico, armonizar el acto docente con la investigación, proyectarse a la sociedad y rendir cuentas son aspectos que deben tomarse en cuenta al momento de definir y poner en práctica un modelo educativo sobre la base de un curriculum integral.

Una base objetiva a los cambios cualitativos que implica una reforma académica o bien el impulso de un modelo educativo en función del desarrollo de la persona, ha de partir de una caracterización de la situación actual que incide en el diseño de un curriculum.

La educación, sobre todo la educación superior no ha sabido articular la práctica docente con la investigación y la vinculación con la sociedad, predominando el acto docente como una actividad que se circunscribe a brindar información en la que generalmente se acude al expediente de la libertad académica con la cual los docentes han asumido toda la responsabilidad de lo que se va a enseñar. Al privilegiar lo tecnológico sobre lo cognitivo se impulsa un modelo curricular, para formar un ciudadano mecanizado autómata que poco o nada promueve la creatividad, el análisis, la comprensión o la reflexión crítica, de ese modo el curriculum se ha concebido solo en aquello que es palpable, básicamente en los planes y programas, descuidando el campo problemático e integral que implica. EI docente ha dejado de ser un elemento activo de la práctica epistemológica para convertirse solo en un ejecutor del curriculum, ello ha contribuido a que los modelos curriculares que se han implantado se hayan visto como algo difuso que no ha partido de un verdadero diagnóstico en la que participen todos los actores principalmente los académicos.

Es así que para un planteamiento curricular como fundamento de cualquier modelo educativo, es bueno partir de la idea de que la educación es una ciencia en torno a la cual se tienen distintas percepciones, que constituye una práctica social y se da en un contexto histórico que implica el diseño de un curriculum que prepare al individuo para insertarse positivamente a un mundo de incertidumbre, lo cultive en valores y asuma la educación como una función continua y 
permanente que nos debe enseñar a convivir y no solo a vivir.

Planteados esos elementos, entre otros que reflejan la problemática curricular, principalmente de la educación superior se postulan ahora algunos aspectos que pueden considerarse en un nuevo modelo de educación, los cuales no están necesariamente acabados:

- Diseñar, construir y ejecutar un curriculum integral que incluya los contenidos conceptuales, contenidos procedimentales y contenidos actitudinales.

- Armonizar la formación técnica con la formación humana en función de los intereses colectivos, concibiendo la educación como generadora de procesos humanos que permita conocer y transformar la realidad para lograr el bien común

- $\quad$ Concebir la educación como un proceso de construcción social que se mide y cuantifica pero además implica significados que se construye desde la práctica.

- Incidir en el estudiante para que basado en el conocimiento de la realidad observable, que no solo le permita describirla, sino también cuestionarla mediante la reflexión crítica para luego emprender la acción transformadora.

E\&A IIES

- En todos los elementos sobre los cuales podemos ver el curriculum, deben tener participación en su estructuración técnica, todos los protagonistas del quehacer académico para generara una conciencia favorable a los cambios que un nuevo modelo ha de implicar.

- $\quad$ Considerando que la práctica docente funciona no solo como una estructura de tareas en donde interactúan maestros y alumnos, sino también como una estructura histórica, el modelo curricular visto desde la acción debe propender a que el profesor además de entrenarse en métodos y técnicas de enseñanza también debe prepararse en la toma de decisiones para lograr el éxito del proceso educativo.

- Los profesores de las distintas disciplinas para que sean protagonistas de la práctica epistemológica, deben ser teóricos y prácticos del curriculum, incorporándose activamente en su diseño, construcción y ejecución que amplíe su capacidad creadora.

- Entendida la educación como un proceso que permite al individuo tener un sentido de colaboración y solidaridad, el diseño, modelo curricular debe 
tomar en cuenta el papel que ha de jugar el individuo frente a la globalidad, la individualidad, la solidaridad, la incertidumbre, la especialización, la complejidad, etc.

- Para responder a las exigencias de la sociedad y a la percepción que ésta tiene sobre la educación, hay que evaluar el curriculum, para valorar los alcances de los programas académicos e institucionales que lleven a tomar las decisiones adecuadas en torno a lo que se hace y sucede, descartando toda sensación de amenaza o descalificación.

Sobre la base de esos elementos y en función de un nuevo modelo educativo que signifique un rediseño curricular e indique qué somos, qué tenemos y hacia dónde queremos llegar, debe ser coherente con el tipo de ciudadano que deseamos formar, para una sociedad más justa, más segura en donde la equidad y la interdependencia como factores integradores sean los signos fundamentales para la paz y la convivencia armónica. 


\section{REFERENCIAS}

Alliud, Andrea y Duschatzky, Laura. Maestros: Formación práctica y transformación escolar. Miño y Davila Editores, Buenos Aires, Argentina 1998.

Albuquerque, Eugenio. Cambio social ¿cambio moral? Ediciones San Pablo, Madrid, España 2001

Benno Sander. Gestión educativa en América Latina: Construcción y reconstrucción del conocimiento. Editorial Troquel, Buenos Aires. 1996

Beard Ruth. Pedagogía y didáctica de la enseñanza universitaria. Oiks-tau, S.A. ediciones. Barcelona, España 1974

Díaz Barriga, Frida; Hernández Rojas Gerardo; Estrategias Docentes para un aprendizaje significativo: una interpretación constructivista. Mc Graw-Hill, México, 1998.

Dierckxsens, Win. El movimiento social por una alternativa al neoliberalismo y a la guerra. Revista centroamericana de economía $n^{\circ} 59-60$. Postgrado centroamericano en economía y planificación del desarrollo UNAH

Espino Cedeño, Maximino; para pensar y hacer la educación. Panamá, 1996

Gómez Buendía Hernández (Director). Educación la agenda del siglo XXI: hacia un desarrollo humano. Programa de las naciones unidas para el desarrollo. PNUD. TM editores. 1998

Hinkelammert, Franz. El asalto al poder mundial y la violencia sagrada del imperio. Colección economía-teología. San José, Costa Rica, 2003

Peñaloza, Walter, snpi

Pérez Lindo, Augusto. Universidad, política y sociedad. Editorial universitaria de Buenos Aires.

Sierra Bravo Restituto. Técnicas de investigación social: teoría y ejercicios, editorial paraninfo, segunda edición, Madrid 1979.

La educación encierra un tesoro. El personal docente en busca de nuevas perspectivas. Cap. 7, ediciones UNESCO. 1997

Confederación de educadores americanos CEA. La responsabilidad social de impulsar, propiciar y defender las políticas de profesionalización plena de los maestros. Cuaderno de trabajo ${ }^{\circ}{ }^{\circ}$. México febrero 1997

E\&A

IIES 
Documento de política para el cambio y el desarrollo en la educación superior. Organización de las naciones unidas para educación, la ciencia y la cultura UNESCO.

Pastoral Universitaria: presencia de la iglesia en la universidad y en la cultura universitaria

Principios de economía micro: económica de la educación snpi 